\title{
Ibuprofen enhances the anticancer activity of cisplatin in lung cancer cells by inhibiting the heat shock protein 70
}

\author{
H Endo ${ }^{1,2}, M_{\text {Yano }}{ }^{*, 1,2}$, Y Okumura ${ }^{1}$ and $\mathrm{H} \mathrm{Kido}^{1}$
}

Hsp70 is often overexpressed in cancer cells, and the selective cellular survival advantage that it confers may contribute to the process of tumour formation. Thus, the pharmacological manipulation of Hsp70 levels in cancer cells may be an effective means of preventing the progression of tumours. We found that the downregulation of Hsp70 by ibuprofen in vitro enhances the antitumoural activity of cisplatin in lung cancer. Ibuprofen prominently suppressed the expression of Hsp70 in A549 cells derived from lung adenocarcinoma and sensitized them to cisplatin in association with an increase in the mitochondrial apoptotic cascade, whereas ibuprofen alone did not induce cell death. The cisplatin-dependent events occurring up- and downstream of mitochondrial disruption were accelerated by treatment with ibuprofen. The increase in cisplatin-induced apoptosis caused by the depletion of Hsp70 by RNA interference is evidence that the increased apoptosis by ibuprofen is mediated by its effect on Hsp70. Our observations indicate that the suppression of Hsp70 by ibuprofen mediates the sensitivity to cisplatin by enhancing apoptosis at several stages of the mitochondrial cascade. Ibuprofen, therefore, is a potential therapeutic agent that might allow lowering the doses of cisplatin and limiting the many challenge associated with its toxicity and development of drug resistance. Cell Death and Disease (2014) 5, e1027; doi:10.1038/cddis.2013.550; published online 30 January 2014

Subject Category: Cancer

The human Hsp70 family includes $\geq 8$ highly homologous members that differ from each other by their intracellular localization and expression patterns. ${ }^{1}$ Among them, the major stress-inducible Hsp70 (also called Hsp72) has an essential role in cell survival under stressful conditions. Compared with its normal counterpart, Hsp70 is often overexpressed in various cancer cells and is suspected to contribute to the development of tumours. ${ }^{2,3}$ Indeed, the expression of Hsp70 in certain cancer types has been correlated with poor prognosis and resistance to chemotherapy. ${ }^{4-6}$

Tumour cells often express several proteins that, when abnormally elevated, render the tumour resistant to apoptosis. $^{7}$ Previous studies have confirmed not only that Hsp70 is cytoprotective, but also that it interferes effectively with cell death induced by a wide variety of stimuli, including several cancer-related stresses. Hsp70 is a potent inhibitor of the stress-activated kinase pathway, and apparently blocks apoptotic signals via interactions with JNK, Ask1 and SEK $1{ }^{8-11}$ Hsp70 is also a negative regulator of the mitochondrial pathway of apoptosis. Much of the focus on the antiapoptotic function of Hsp70 has been on events that occur after the disruption of the mitochondria. Hsp70 prevents the recruitment of procaspase- 9 to the apoptosome, and its functional complex formation by direct interaction with apoptotic protease-activating factor 1 (Apaf-1). ${ }^{12,13}$ Furthermore, Hsp70 inhibits the activation of caspase-3 and the cleavage of caspase- 3 targets, such as ICAD and GATA-1. ${ }^{14,15}$ On the other hand, recent studies have reported that Hsp70 can prevent apoptosis upstream of the mitochondria, by inhibiting events, which, ultimately, permeabilize the mitochondrial outer membrane, such as the activation of Bax. ${ }^{16,17}$

As a result of the inhibition by Hsp70 of the apoptosis induced by several anticancer drugs, as well as by other stimuli, we hypothesized that cancer cells would be sensitized to the induction of apoptosis by the neutralization of Hsp70. Hsp70 has been, indeed, targeted with pharmaceuticals, such as triptolide, quercetin and KNK437, which downregulate its expression. ${ }^{18-20}$ Although they have prevented the progression of various cancer cells in vitro and in vivo, ${ }^{21,22}$ the optimal clinical use of these small Hsp70 inhibitors singly or combined with other chemotherapeutics remains a challenge. Our overall objective was to pharmacologically control the levels of Hsp70, and increase the effectiveness of anticancer drugs.

Several experimental and epidemiologic studies and clinical trials have observed a powerful chemopreventive activity exerted by nonsteroidal anti-inflammatory drugs (NSAIDs). ${ }^{23,24}$ The anti-carcinogenic properties of NSAID

\footnotetext{
${ }^{1}$ Division of Enzyme Chemistry, Institute for Enzyme Research, The University of Tokushima, Tokushima, Japan and ${ }^{2}$ Department of Nutrition, School of Human Cultures, The University of Shiga Prefecture, Shiga, Japan

${ }^{*}$ Corresponding author: M Yano, Department of Nutrition, School of Human Cultures, The University of Shiga Prefecture, Hikone, Shiga 522-8533 Japan. Tel: +81 74928 8441; E-mail: yano.m@shc.usp.ac.jp

Keywords: Hsp70; apoptosis; ibuprofen

Abbreviations: Apaf-1, apoptotic protease-activating factor 1; COX, cyclooxygenase; HSF-1, heat shock factor 1; MTT, 3-(4,5-dimethylthiazol-2-yl)-2, 5-diphenyltetrazolium bromide; NSAID, nonsteroidal anti-inflammatory drug; NSCLC, non-small cell lung cancer; PCR, polymerase chain reaction; RNAi, RNA interference; RT, reverse transcriptase; TUNEL, terminal deoxynucleotidyl transferase-mediated dUTP nick and labelling

Received 25.6.13; revised 27.11.13; accepted 10.12.13; Edited by G Raschellà
} 
have been attributed to their inhibition of cyclooxygenase (COX) enzymes. However, much higher doses of NSAID are needed to obtain an antitumoural effect than to inhibit COX, ${ }^{25}$ suggesting that they also act via COX-independent mechanisms. On the other hand, NSAIDs, such as aspirin, salicylate and sulindac sulphide, inhibit the proliferation of cells and induce apoptosis in various cancer cell lines, which is considered an important component of their antitumoural activity, and increased sensitization of cancer cells to anticancer drugs. ${ }^{26-29}$ There is currently interest in the ability of NSAID to directly lower the levels of antiapoptotic molecules, such as the Bcl-2 family ${ }^{30}$ and 14-3-3 protein, ${ }^{31}$ which inhibits the intrinsic mitochondria-dependent apoptosis in various cancer cells. Therefore, the NSAID-induced dysfunction of antiapoptotic proteins prompted us to examine whether other antiapoptotic molecules, including Hsp70, might also be targets in the prevention of tumour progression by NSAID. In this study, we show that ibuprofen is a potent inhibitor of Hsp70, which significantly suppresses its expression by depleting heat shock factor 1 (HSF1) in lung adenocarcinoma-derived A549 cells. The downregulation of Hsp70 by ibuprofen sensitized the cells to cisplatin, which was associated with the enhancement of cisplatin-induced apoptotic signalling. Ibuprofen did not only facilitate postmitochondrial events, including the activation of cisplatin-induced caspase-9, but also the activation of Bax, causing the release of cytochrome $c$. Besides the demonstration of a similar increase in the sensitivity of A549 cells to cisplatin conferred by Hsp70 knockdown and ibuprofen, these observations indicate that ibuprofen accelerates cisplatin-mediated apoptosis at multiple steps of the mitochondrial apoptotic pathway via the inhibition of Hsp70. We conclude that ibuprofen is a potential chemotherapeutic agent, which might enable (a) the use of lower, less toxic does of cisplatin and (b) the design of a new combination treatment of lung cancer.

\section{Results}

Ibuprofen suppresses the expression of Hsp70 in lung adenocarcinoma cells. To define the role of $\mathrm{Hsp} 70$ in promoting the formation of tumours, we first examined its expression in human lung cancer cell lines. Compared with BEAS-2B, a human, non-malignant, bronchial epithelial cell line, the expression levels of Hsp70 in lung cancer cells, such as A549 and H358 adenocarcinoma, were notably higher (Figure 1a). As in previous studies, which showed an increased expression of Hsp70 in various types of human cancers, including breast, pancreas and colon, we found that Hsp70 is also dysregulated in lung cancer cells. In this study, we screened conventional NSAID in search of a new pharmacologic inhibitor, which neutralizes Hsp70, as they induce apoptosis in cancer cells by selectively downregulating antiapoptotic proteins. The expression of Hsp70 after the exposure of A549 cells to various NSAID in non-toxic concentrations, was analyzed by immunoblot. Ibuprofen, in a $400-\mu \mathrm{M}$ concentration, decreased the expression of Hsp70 by $23 \%$ in comparison with untreated cells, whereas other NSAID had no effect (Table 1). Figure $1 \mathrm{~b}$ shows the decrease in Hsp70 protein and mRNA levels in A549 cells, after treatment with various concentrations of ibuprofen, versus no apparent decreases in $\mathrm{Hsc70}$ and Actin. Ibuprofen also decreased the expression of Hsp70 in H358, a human lung adenocarcinoma cell line, in a dose-dependent manner (Figure 1c). These results suggest that ibuprofen decreases the expression of Hsp70 in various lung cancer cell lines.

Ibuprofen enhances the apoptosis induced by cisplatin by suppressing Hsp70. As ibuprofen prominently inhibited the expression of Hsp70, we next examined its effect on the proliferation of cancer cells. We observed no significant change in the viability of A549 and H358 cells after the

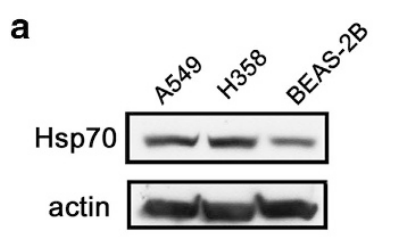

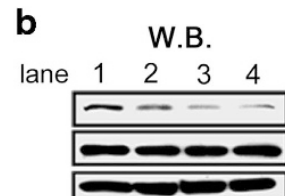
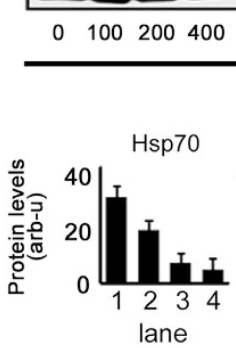

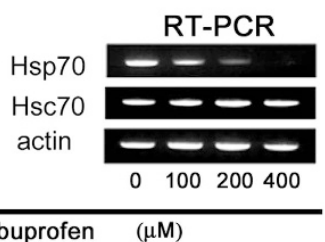

lbuprofen $\quad(\mu \mathrm{M})$

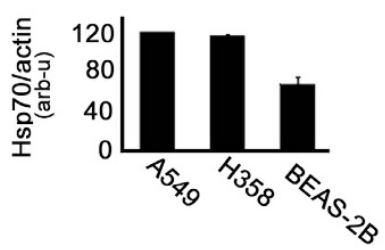

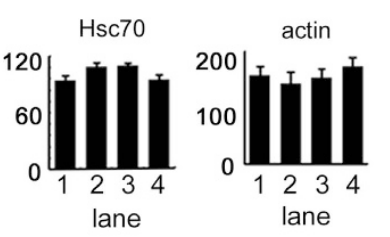

C
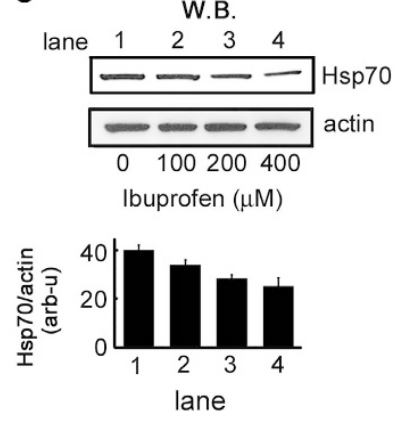

Figure 1 lbuprofen suppresses the expression of Hsp70 in lung adenocarcinoma cells. (a) Upregulation of Hsp70 in lung cancer cell lines. Each cell extract was separated by SDS-PAGE and immunoblotted with an anti-Hsp70 or actin antibody (upper panel). The quantity of each protein was estimated by densitometric analysis using Scion Image (Scion, Frederick, MD, USA). The Hsp70/actin ratios are shown in the lower panel. (b) Effect of ibuprofen on the expression of Hsp70 protein and mRNA in A549 cells. Top: expression of Hsp70 and Hsc70 proteins in A549 cells treated with ibuprofen at the specified concentrations for $48 \mathrm{~h}$, examined as described in a (left panel). Bottom: densitometric analysis of each protein level in arbitrary unit (arb-u). The alternation of each mRNA expression after ibuprofen treatment was analyzed by semiquantitative RTPCR (top right panel). These results are representative of three separate experiments. (c) Effect of ibuprofen on the expression of Hsp70 protein in $\mathrm{H} 358 \mathrm{cells}$. Expression of $\mathrm{Hsp} 70$ proteins in $\mathrm{H} 358$ cells treated with ibuprofen at the specified concentrations for $48 \mathrm{~h}$ (upper panel). The quantity of each protein was estimated by densitometric analysis (lower panel) 
Table 1 Effects of nonsteroidal anti-inflammatory drugs on the expression of Hsp70 in A549 cells

\begin{tabular}{lc}
\hline NSAIDs & Hsp70 expression (\%) \\
\hline Ibuprofen $(400 \mu \mathrm{M})$ & $22.7 \pm 2.8$ \\
Aspirin $(2500 \mu \mathrm{M})$ & $95.1 \pm 7.8$ \\
Diclofenac $(200 \mu \mathrm{M})$ & $97.2 \pm 5.6$ \\
Sulindac $(15 \mu \mathrm{M})$ & $98.9 \pm 2.9$ \\
Piroxicam $(60 \mu \mathrm{M})$ & $96.6 \pm 6.2$ \\
Indometacin $(10 \mu \mathrm{M})$ & $95.0 \pm 15.1$ \\
Mefenamic acid $(25 \mu \mathrm{M})$ & $100.5 \pm 6.0$ \\
\hline
\end{tabular}

Values are shown as means \pm S.D.

The expression of $\mathrm{Hsp} 70$ was measured by immunoblotting with an anti-Hsp70 antibody. The quantity of Hsp70 protein was estimated by densitometric analysis using Scion Image. The values in parentheses are the highest nontoxic concentrations (approximately $90 \%$ viability) used for each NSAID on the A549 cells for $48 \mathrm{~h}$

exposure to $\sim 800 \mu \mathrm{M}$ concentrations of ibuprofen alone, which downregulates Hsp70 (Figure 2a), while the exposure to $1.0 \mathrm{mM}$ concentration of ibuprofen caused cell death. Combined, these observations indicate that the downregulation of stress-inducible Hsp70 was insufficient to cause the death of A549 and H358 cells.

There is evidence that the inhibition of anti-apoptotic molecules, such as Hsp70, increases the sensitivity of tumour cells to anticancer drugs, thus improving the outcomes of chemotherapy. To study the therapeutic potential of ibuprofen, we examined whether its antitumoural effects are synergistic with those of cisplatin widely used in the treatment of lung adenocarcinoma. When we measured the survival of A549 (top of Figure 2b) and H358 (bottom of Figure 2b) cells exposed to increasing concentrations of cisplatin incubated in presence versus absence of ibuprofen, the latter prominently magnified the apoptosis induced by cisplatin, a synergistic effect confirmed by terminal deoxynucleotidyl transferase-mediated dUTP nick and labelling (TUNEL) staining (Figure 2c). To ascertain the effects conferred by the expression of Hsp70 on cell death, while excluding all effects of ibuprofen unrelated to Hsp70, we weakened the expression of Hsp70 by RNA interference (RNAi) (Figure 2d) and measured its effects on the apoptosis induced by cisplatin. The inhibition of Hsp70 decreased the viability of cisplatin-treated cells by approximately $20 \%$ (Figure $2 \mathrm{e}$ ). Transfections with scrambled siRNA, serving as a control, showed no increase in cell death mediated by cisplatin. Cisplatin had no effect on the expression of Hsp70 (Figure 2g). We quantified the number of apoptotic cells in ibuprofen- and/or cisplatin-treated cultures using the CF488A-annexin $\mathrm{V}$ methods. Although cisplatin alone induced apoptosis in $10.2 \%$ of A549 cells, the co-treatment with ibuprofen increased the percentage of apoptotic cells to $34.0 \%$ (Figure 2f). These observations suggest that ibuprofen sensitizes A549 cells to cisplatin by decreasing the expression of Hsp70.

Ibuprofen decreases the expression of Hsp70 via transcriptional inactivation. The reverse transcriptasepolymerase chain reaction (RT-PCR) analysis described earlier revealed a decrease in RNA level following treatment with ibuprofen, suggesting that the expression of Hsp70 can be downregulated at the transcriptional level. After the recently discovered inhibition, by its antagonists, of the transcription of $\mathrm{Hsp} 70$ in cancer cells by blockade of the activation of $\mathrm{HSF}^{18,20}$ (which is often upregulated and constitutively activated in tumour formation), we studied the effects of ibuprofen on HSF1 in A549 cells. We first performed a ChIP assay to explore whether the inhibitory effect of ibuprofen is at the level of HSF1 DNA binding. As expected, we found an unequivocal association between HSF1 and the Hsp70 gene promoter containing the HSE site, in ibuprofen-untreated cells (Figure 3a). It is noteworthy that ibuprofen eliminated this binding (Figure 3a), suggesting that it inhibits the expression of Hsp70 via the action of HSF1. This also suggests that ibuprofen blocks the binding of HSF1 chromatin, or the steps which precede, in several processes needed to activate HSF1. Therefore, we broadened our analysis to examine the effect of ibuprofen on the expression of HSF1. Compared with unexposed, control cells, the HSF1 mRNA level was significantly lower in cells exposed to ibuprofen (bottom of Figure 3b). Consistent with its effect on the expression of mRNA, ibuprofen also decreased the expression of HSF1 protein in a dose-dependent fashion (top of Figure $3 b$ ). To confirm the inhibition of HSF1mediated Hsp70 by ibuprofen, we lowered the amounts of HSF1 present in A549 cells by RNAi, and studied its effect on the expression of Hsp70. The treatment of cells with HSF1 dsRNA decreased the Hsp70 level compared with that measured in cells untreated with dsRNA (Figure 3c). Ibuprofen decreased the expression of HSF1 by $16 \%$ in comparison with untreated cells, whereas other NSAID had no effect (Table 2). Overall, these observations indicate that ibuprofen inhibited the expression of Hsp70 by depleting the HSF1 in A549 cells.

Ibuprofen accelerates the mitochondrial apoptotic process induced by cisplatin. Several studies have found that mitochondria might be a direct and important target of cisplatin in sensitive cells. ${ }^{32,33}$ We studied the effects of ibuprofen on the depolarization of mitochondrial membranes and the cytochrome $c$ release induced by cisplatin. A549 cells with or without cisplatin were incubated in absence or presence of ibuprofen and stained with JC-1. Treatment with cisplatin and ibuprofen lowered the mitochondrial membrane potential, manifest by an attenuated red and an enhanced green mitochondrial fluorescence (Figure 4a, lower right panel) compared with that observed with cisplatin alone (Figure 4a, upper right panel), while control (Figure $4 a$, upper left panel) or ibuprofen alone (Figure 4a, lower left panel) produced the red-dotted staining pattern of polarized mitochondria. The intensity of green mitochondrial fluorescence in cisplatin-treated cells is significantly increased (36.56 to $55.56 \%$ ) by the co-treatment with ibuprofen. Ibuprofen also promoted the release of cytochrome $c$ from the mitochondria induced by cisplatin (Figure $4 \mathrm{~b}$ ). These findings unequivocally indicated that, in A549 cells, ibuprofen enhanced the mitochondria-dependent apoptosis caused by cisplatin.

Ibuprofen increases the activation of Bax induced by cisplatin. The translocation of the pro-apoptotic protein Bax 
to the mitochondria is closely associated with the apoptosis induced by cisplatin. To explore the mechanisms by which ibuprofen promotes the apoptosis mediated by mitochondria in response to cisplatin, we examined whether it was due to its ability to stimulate the translocation of Bax by cisplatin. We first monitored conformational changes in Bax as indicators of its activation. Western blot analysis of the immunoprecipitates with a conformation specific anti-Bax (6A7) antibody, which only recognizes the active form, revealed the presence of active Bax in A549 cells treated with cisplatin (Figure 5a, lane 4), although not in untreated cells (Figure $5 \mathrm{a}$, lanes 1 and 2 ). Further exposure of the
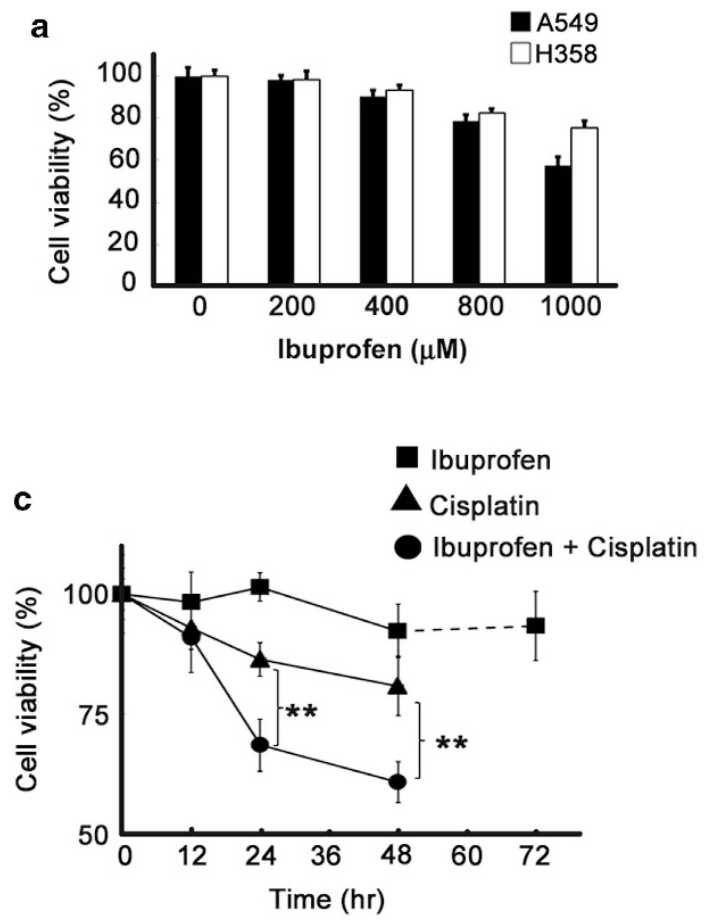
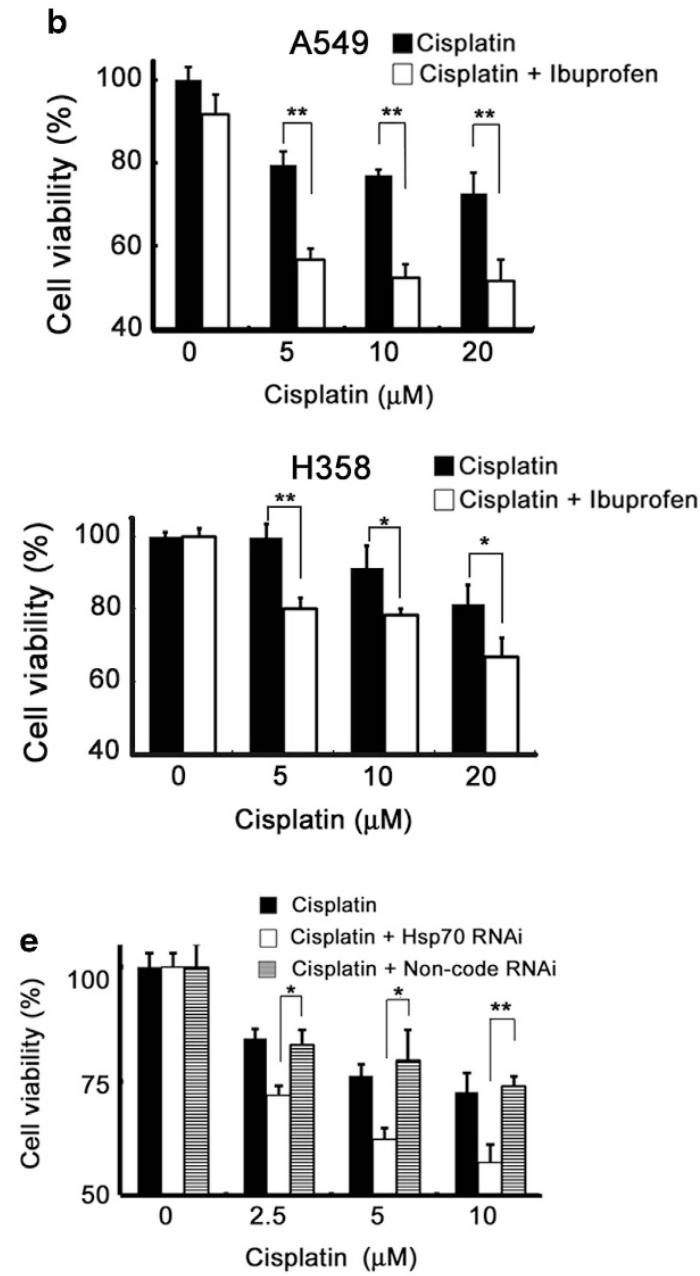

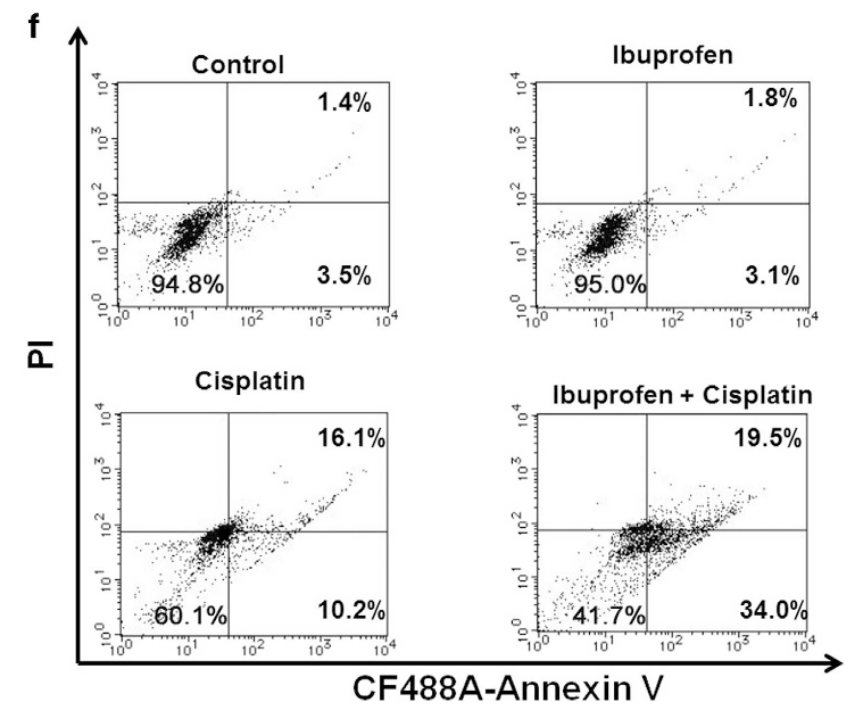

g

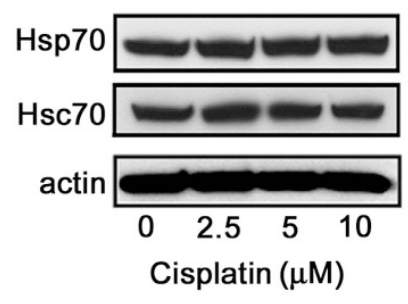


cisplatin-treated cells to ibuprofen caused a 1.5-fold increase in active Bax, compared with incubation with cisplatin alone (Figure 5a, lane 3). When we analyzed the effects conferred by ibuprofen on the translocation of Bax to mitochondria in cisplatin-treated cells, we observed an approximately 1.3-fold increase in the amount of translocated Bax (Figure 5b). To exclude an effect of ibuprofen unrelated to the inhibition of Hsp70, we performed RNAi for a selective knock-down of Hsp70, and we studied its effects on the activation of Bax. Consistent with the earlier data presented for ibuprofen, the depletion of Hsp70 increased the activation of Bax in cisplatin-treated cells, although its extent was greater with Hsp70 RNAi than with ibuprofen (Figure 5c). These observations confirmed that (a) ibuprofen promotes the activation of Bax dependent on cisplatin and its translocation to the mitochondria in A549 cells and (b) its mechanism of action is mediated by the inhibition of Hsp70.

Ibuprofen facilitates events occurring upstream and downstream of mitochondrial disruption in cisplatinmediated apoptosis. Previous studies have shown that Hsp70 can inhibit apoptosis by acting downstream of the mitochondria. $^{12-15}$ Hsp70 interacts directly with Apaf-1 to prevent the formation of cytochrome $c$-mediated apoptosome and subsequent activation of caspase-9. To examine whether ibuprofen also influences the downstream mitochondrial events, we measured its effects on the cleavage of procaspase- 9 in the apoptosis mediated by cisplatin. With an anti-active caspase- 9 antibody, fully processed caspase- 9 was predominantly identified in cisplatin-treated A549 cells

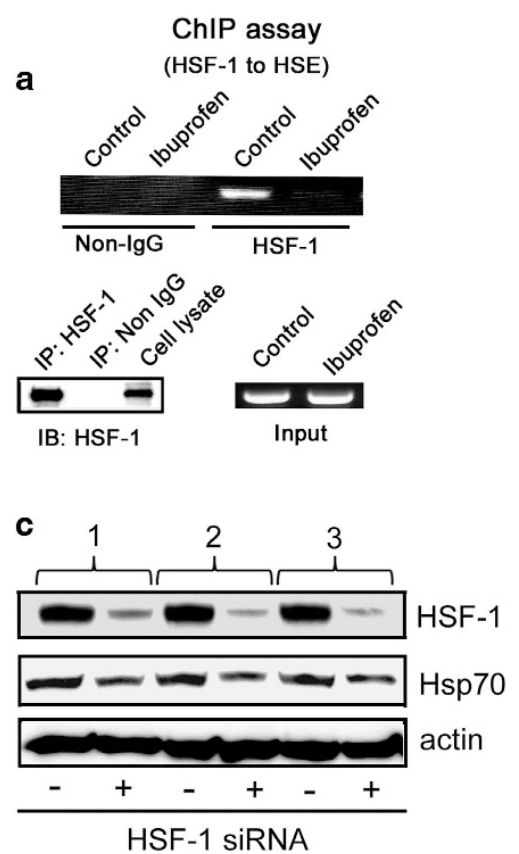

b
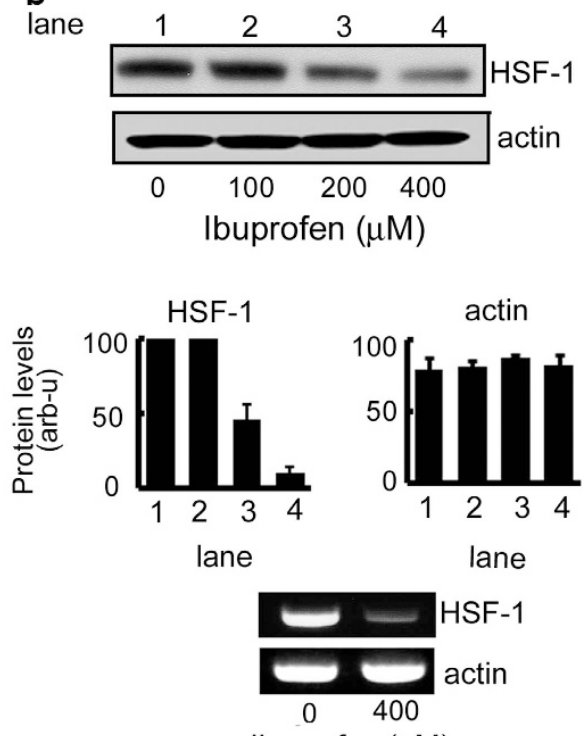

Ibuprofen $(\mu \mathrm{M})$

Figure 3 Ibuprofen inhibits the expression of Hsp70 by transcriptional inactivation. (a) ChIP assay for the association of HSF-1 with Hsp70 gene in A549 cells treated with or without ibuprofen. The DNA in the product immunoprecipitated by anti-HSF-1 or non-immune IgG was followed by PCR with a primer specific to the Hsp70 promoter. The immunoprecipitates with antibody against HSF-1 were confirmed by immunoblotting (bottom left). The actin signal is a control of DNA input (bottom right). (b) Effect of ibuprofen on the expression of HSF-1. Cell extracts from A549 cells with ibuprofen for $48 \mathrm{~h}$ were separated by SDS-PAGE and immunoblotted with a HSF-1-specific antibody (upper panel). The quantity of each protein was estimated by densitometric analysis (middle panel). The data are representative of three separate experiments. The effect of ibuprofen on the mRNA level of HSF-1 was confirmed by RT-PCR (lower panel). (c) HSF1-mediated inhibition of Hsp70 expression. A549 cells were treated with 10 nM siRNAs against HSF-1 or non-code siRNA. The HSF-1 silencing efficiency and its effect on the expression of Hsp70 were examined by immunoblotting, using appropriate antibodies. Three separate siRNAs oligo against HSF-1 were used for its knock-down. The results shown are representative of three separate experiments

Figure 2 Ibuprofen increased the antitumoural activity of cisplatin by suppressing Hsp70. (a) The viability of A549 (upper panel) and H358 (lower panel) cells treated with ibuprofen for $48 \mathrm{~h}$ was analyzed by MTT assay. The value is represented as the percentage of cell viability without ibuprofen, set at $100 \%$. (b) Synergistic effect of ibuprofen on cisplatin-induced apoptosis in A549 (upper panel) or H358 (lower panel) cells. The cells were treated with the specified concentrations of cisplatin in absence or presence of $400 \mu \mathrm{M}$ ibuprofen for $48 \mathrm{~h}$, and the cell viability was assessed by MTT assay. The results are shown as means \pm S.D. from triplicated experiments. The results shown are representative of three separate experiments. ${ }^{*} P<0.05 ;{ }^{*} P<0.01$ (by Student's $t$-test). (c) Time course of cisplatin-mediated cell death with ibuprofen. A549 cells were treated with $400 \mu \mathrm{M}$ ibuprofen alone or A549 cells with $10 \mu \mathrm{M}$ cisplatin were cultured in absence or presence of $400 \mu \mathrm{M}$ ibuprofen, and the cell viability was analyzed by TUNEL staining. The results shown as means \pm S.D. ${ }^{* \star} P<0.01$ (by Student's $t$-test). (d) The silencing efficiency of Hsp70 determined by immunoblotting. (e) Effect of Hsp70 RNAi on the cisplatin-mediated death of A549 cells. The cells exposed to siRNA targeting Hsp70 or control siRNA were treated with $10 \mu \mathrm{M}$ cisplatin for $48 \mathrm{~h}$, and MTT assay was used to determine the cell viability. Data are presented as means \pm S.D. from triplicated experiments. The results shown are representative of three separate experiments. ${ }^{*} P<0.05$; ${ }^{*} P<0.01$ (by Student's $t$-test). (f) Cytofluorimetric dot plot analysis of the CF488A-Annexin V versus propidium iodide (PI) staining performed in $10 \mu \mathrm{M}$ cisplatintreated or -untreated A549 cells in absence or presence of $400 \mu \mathrm{M}$ ibuprofen for $48 \mathrm{~h}$. A representative experiment out of three performed with similar results is shown. (g) Effect of cisplatin on the expression of Hsp70. The data are representative of three separate experiments 
(Figure 6a, lane 3) over untreated cells (Figure 6a, lanes 1 and 2). It is noteworthy that treatment with ibuprofen increased $>4$-fold the amount of active caspase- 9 in cells treated with cisplatin, compared with cells incubated with cisplatin alone (Figure 6a, lane 4). As, as reported earlier, the highest increases in the activation of Bax and release of cytochrome $c$ by ibuprofen were $<2$-fold, these observations suggest that ibuprofen also facilitates the post mitochondrial process taking place between the release of cytochrome $c$ and the activation of caspase-9. To verify that this is a specific effect, we studied the effect of Hsp70 knock-down on the activation of caspase- 9 mediated by cisplatin. The caspase- 9 activity in cells depleted of Hsp70 with cisplatin was fourfold greater than in control (scrambled) siRNAtreated cells (Figure $6 \mathrm{~b}$ ). We obtained similar results when we measured the activity of caspase- 9 in cells treated with ibuprofen (Figure 6c) or siRNA against Hsp70 (Figure 6d) by a fluorometric assay using a synthetic substrate.

Table 2 Effects of nonsteroidal anti-inflammatory drugs on the expression of HSF-1 in A549 cells

\section{NSAIDs}

Ibuprofen $(400 \mu \mathrm{M})$

Aspirin $(2500 \mu \mathrm{M})$

Diclofenac $(200 \mu \mathrm{M})$

Sulindac $(15 \mu \mathrm{M})$

Piroxicam $(60 \mu \mathrm{M})$

Indometacin $(10 \mu \mathrm{M})$

Mefenamic acid $(25 \mu \mathrm{M})$
HSF-1 expression (\%)

$16.2 \pm 3.9$

$93.5 \pm 2.9$

$96.7 \pm 6.6$

$99.8 \pm 3.6$

$96.3 \pm 4.7$

$98.1 \pm 1.6$

$98.5 \pm 1.1$
Values are shown as means \pm S.D.

The expression of HSF-1 was measured by immunoblotting with anti-HSF-1 antibody. The quantity of HSF-1 protein was estimated by densitometric analysis using Scion Image. The values in parentheses are the highest nontoxic concentrations (approximately $90 \%$ viability) used for each of the NSAID on the A549 cells for $48 \mathrm{~h}$

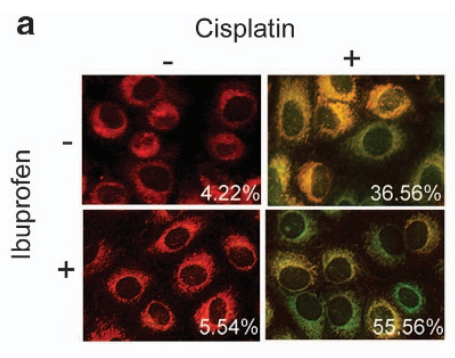

b Cytosolic fraction
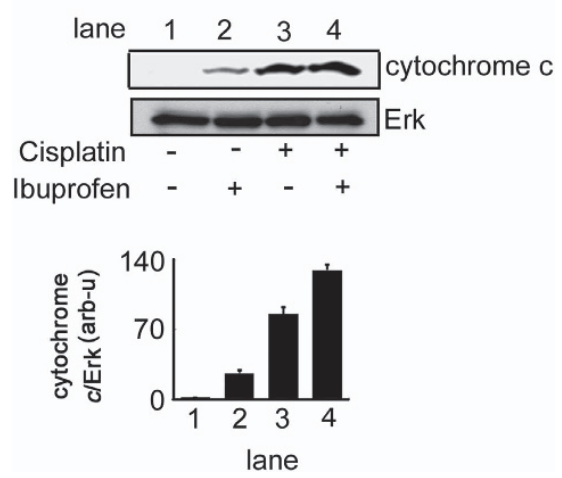

Figure 4 Ibuprofen exposure enhanced the cisplatin-dependent mitochondrial membrane depolarization and cytochrome $c$ release. (a) A549 cells were treated for $48 \mathrm{~h}$ with $10 \mu \mathrm{M}$ cisplatin, $400 \mu \mathrm{M}$ ibuprofen, or both, and subjected to $\mathrm{JC}-1$ staining to study the changes in mitochondrial membrane potential. The percentages indicate the green fluorescence intensity of JC-1 measuring with FACSCalibur. A representative experiment out of three performed with similar results is shown. (b) A549 cells, treated as described earlier, were fractionated into cytosol, and the release of cytochrome $c$ was analyzed by western blot, using anti-cytochrome $c$ antibody. The expression of Erk was monitored as an internal control of cytosol protein. The quantity of each protein was estimated by densitometric analysis. The results are means of three separate experiments from cells in different cultures
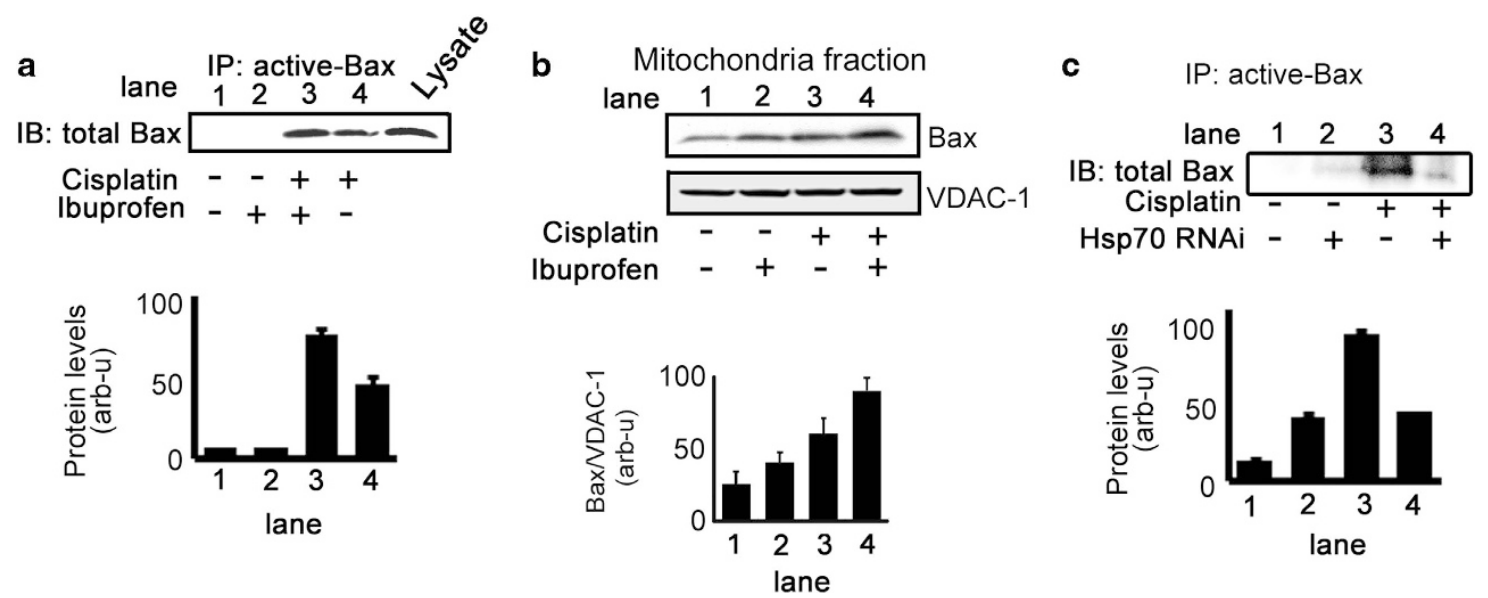

Figure 5 The downregulation of Hsp70 increased the cisplatin-mediated activation of Bax and its translocation to the mitochondria. (a) Detection of active Bax. A549 cells were treated with cisplatin $(10 \mu \mathrm{M})$ and/or ibuprofen $(400 \mu \mathrm{M})$ for $48 \mathrm{~h}$. Active Bax was immunoprecipitated with an active conformation-specific monoclonal antibody and revealed by immunoblotting with an anti-Bax polyclonal antibody. The quantity of active Bax was estimated by densitometric analysis. (b) A549 cells, treated as described earlier, were lysed and fractionated by differential centrifugation to separate the mitochondria from the cytosol. The translocation of Bax to the mitochondria was visualized by the immunoblot of mitochondrial fractions, using an anti-Bax antibody. VDAC-1 was used as a loading control to ensure the use of equal amounts of mitochondria. (c) A decrease in Hsp70 by RNAi promoted cisplatin-dependent activation of Bax. A549 cells treated with Hsp70 or control siRNA were incubated in presence or absence of cisplatin; each cell extract was immunoprecipitated with an anti-active Bax antibody, followed by immunoblotting with anti-Bax antibody. The data are representative of three separate experiments 
a
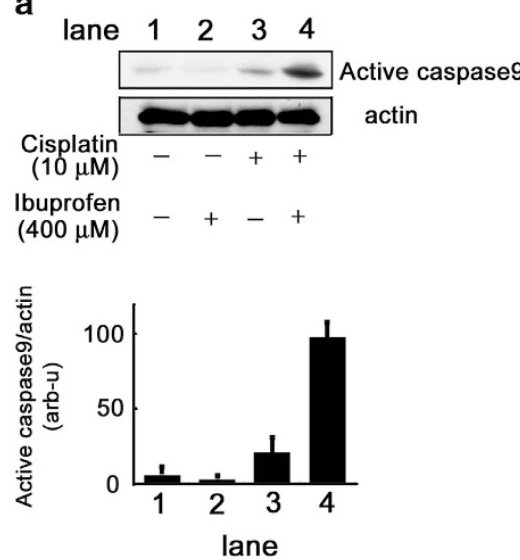

C

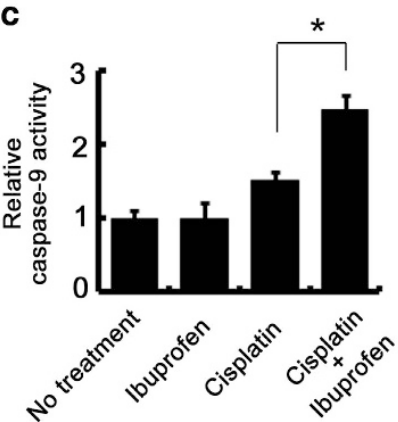

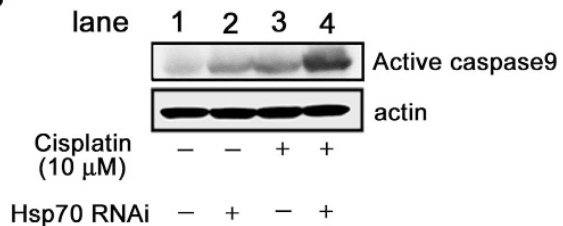

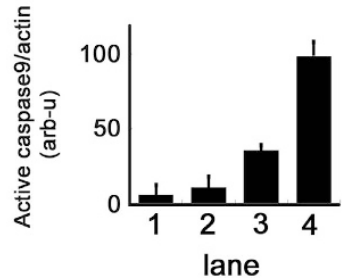

d

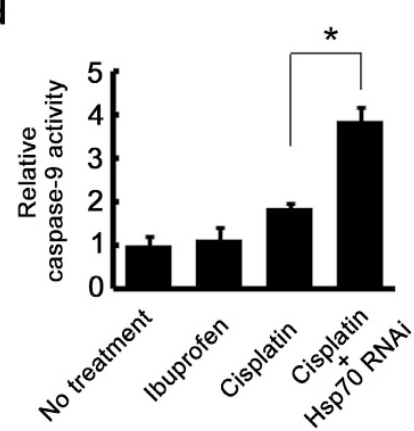

Figure 6 Synergistic effect of Hsp70 suppression on the cisplatin-mediated activation of caspase-9. (a) A549 cells were treated with cisplatin and/or ibuprofen, and cell extracts were immunoblotted with active caspase-9 antibody. The lower panel shows the measurement of each caspase-9. (b) A549 cells exposed to siRNA targeting Hsp70 or control siRNA were incubated with or without cisplatin, and the active caspase-9 was detected by western blot using an anti-caspase-9 antibody. The quantity of each protein was estimated by densitometric analysis (lower panels). (c and d). Assay for enzymatic activity of caspase-9, using a fluorogenic substrate. (c) After the incubation of the A549 cells with cisplatin $(10 \mu \mathrm{M})$ and/or ibuprofen $(400 \mu \mathrm{M})$, the caspase-9 activity of each cell extract was measured as described in Materials and methods section. (d) A549 cells transfected with Hsp70 siRNA or control siRNA were exposed to cisplatin for $48 \mathrm{~h}$. The caspase-9 activity was then assessed, using an enzymatic assay as described earlier. The value of caspase- 9 activity was presented relative to the activity in untreated cells, set at 1.0. The data represent mean values of three separate experiments. Significances were determined by Student's $t$-test $\left({ }^{*} P<0.05\right)$

Overall, these observations confirmed unambiguously that ibuprofen intensified the apoptosis induced by cisplatin by its effects on the events occurring downstream of the mitochondria, by inhibiting Hsp70, although whether it stimulated the formation of apoptosome (essential for the recruitment of procaspase-9) remains to be determined. We conclude that ibuprofen promotes the apoptosis induced by cisplatin at multiple stages of the mitochondrial cascade, by attenuating the expression of Hsp70 in A549 cells.

\section{Discussion}

We found that, compared with non-malignant bronchial epithelial cells, human lung cancer cells overexpressed Hsp70. This is an important observation, as targeting the expression or function of Hsp70 has been suggested as an effective treatment strategy in several cancers, based on the hypothesis that higher levels of Hsp70 protect against cell death and increase the survival rate against modalities used in chemotherapy. ${ }^{11,15}$ In fact, it is well documented that the expression of $\mathrm{Hsp} 70$ is significantly increased in cancer tissues and/or serums obtained from patients with non-small cell lung cancer (NSCLC) $)^{34-38}$ and its overexpression correlates with poor prognosis in NSCLC. ${ }^{36}$ Several reports have indicated that functionally related small molecules that inhibit Hsp70 decrease the viability of colo-rectal or pancreatic cancer cells by promoting apoptosis via the downregulation of Hsp70, and may be a promising new class of cancer chemotherapeutics. ${ }^{19,21,22}$ We showed that ibuprofen, a relatively non-toxic and widely used NSAID, significantly decreased the expression of Hsp70 in lung adenocarcinoma cell lines. We also clearly demonstrated that the inhibitory mechanisms of ibuprofen on $\mathrm{Hsp} 70$ are due to a decrease in HSF1 expression. Although the fundamental mechanism behind the reduction in HSF-1 expression is unknown, a previous study has indicated that the nuclear factor 1 family member NFIX, which codes for site-specific DNA-binding proteins known to have multiple roles in replication, signal transduction and transcription, exerts a transcriptional repressive effect on the expression of HSF1 in cancer cells. ${ }^{39}$ Whether NFIX is, indeed, involved in the inhibition of HSF1 expression evoked by ibuprofen is applicable in further studies. To the best of our knowledge, this is the first study of the inhibitory effects of NSAID on the cellular expression of Hsp70. In addition, we showed that ibuprofen does not influence the cell viability without additional stimuli, unlike its 
maximal effect on the expression of Hsp70. The lack of inhibitory efficacy of ibuprofen against tumours is consistent with a previous study, which showed that low-dose ibuprofen did not induce apoptosis in mouse and human colorectal cancer cell lines. ${ }^{29}$ Similar observations were made following RNAi of Hsp70, suggesting that the attenuation of Hsp70 per se is insufficient to cause the death of A549, and perhaps other cells. It has been shown that the knockdown of Hsp70 has no effect on the viability of several cancer cell lines, although sensitized them to anticancer drugs. ${ }^{40,41}$ Therefore, the therapeutic potential of ibuprofen combined with chemotherapeutic agents needs to be explored.

Cisplatin is one of most effective chemotherapeutic drugs against NSCLCs. ${ }^{42}$ It is noteworthy that damage to DNA caused by cisplatin enables apoptosis involving mitochondrial pathways, which is negatively regulated by Hsp70. As ibuprofen prominently suppressed the expression of Hsp70 in A549 and H358 cells, we examined the possible synergistic activity of ibuprofen and cisplatin against cancer. As expected, ibuprofen potentiated synergistically the anti-proliferative effect of cisplatin in A549 and H358 cells. Despite its potent antitumoural properties, the therapeutic use of cisplatin in oncology is seriously limited by dose-dependent adverse effects and frequent development of drug resistance. ${ }^{43}$ Therefore, our findings may make useful contributions toward the development of new and less toxic chemotherapy against NSCLCs.

We also examined the molecular mechanisms of these synergistic properties of ibuprofen. Hsp70 protects cells against mitochondria-dependent apoptosis at different levels, although the precise mechanism remains hypothetical, because of regular contradictory descriptions of Hsp70 function. Earlier reports have shown a protective effect of Hsp70 against cellular apoptosis by inhibition of the apoptosome function, a protein complex comprising Apaf-1 and cytochrome $c .{ }^{12,13}$ However, recent reports have questioned this repression of apoptosis downstream of the mitochondrial membrane permeabilization. Several studies have suggested that Hsp70 functions upstream of the mitochondria by preventing the release of cytochrome $c$, instead of inhibiting the apoptosome or other downstream points in the caspase cascade. ${ }^{16,17}$ Some of this confusion may be due to different experimental systems used to evaluate apoptosis, or reflects the variability of apoptotic pathways among different cell lines. In this study, we found that the inhibition of Hsp70 by ibuprofen facilitates the activation of Bax induced by cisplatin, and its translocation to the mitochondria in A549 cells. This finding is consistent with the previous observation of blockade of Bax activation being one of the upstream sites of action of Hsp70. On the other hand, the role played by Hsp70 in the A549 cellular mitochondrial apoptotic pathway is likely to be more complex than described earlier because, in cisplatin-treated cells, the decreased expression of $\mathrm{Hsp} 70$ caused by ibuprofen amplified the activation of caspase- 9 significantly compared with that of Bax. Furthermore, the similar increase in the activation of cisplatin-dependent Bax and release of cytochrome $c$ by ibuprofen suggests that Hsp70 also inhibits the post mitochondrial steps between the release of cytochrome $c$ and the activation of caspase-9. If Hsp70 were acting downstream of the mitochondria, one would predict that it interferes with the activation of caspase- 9 in response to cytochrome $c$, either by inhibiting the formation of the apoptosome, or by preventing the binding of pro-caspase- 9 to this complex. When we studied the effects of Hsp70 on the formation of, and recruitment of pro-caspase- 9 to, the apoptosome, the cell lysates were immunoprecipitated, although Hsp70 failed to migrate with Apaf-1, cytochrome $c$ or caspase-9 (data not shown). These results may be supported by previous report that no association between Hsp70 and Apaf-1 or apoptosome complex was observed, even under in vitro activation of caspase by the addition of cytochrome $c$ and dATP. ${ }^{44}$ Furthermore, we were unable to identify a new target for Hsp70 in the process of caspase- 9 activation, indicating that its inhibitory activity is attributable to another indirect effect, instead of a direct one as previously reported. Altogether, the data presented here are the first evidence of cell death inhibition by Hsp70, by its targeting of both upstream and downstream mitochondrial processes, while the precise mechanisms by which it interferes with the activation of caspase- 9 remains to be clarified.

In conclusion, ibuprofen potentiated the antitumoural properties of cisplatin in the cells of lung adenocarcinoma, via a mechanism of action mediated by the suppression of Hsp70. These findings may promote the development of a new strategy to increase the effectiveness of cisplatin in the treatment of NSCLCs, as well as highlight the putative merits of developing anticancer treatments targeting Hsp70.

\section{Materials and Methods}

Materials. The mouse monoclonal anti-Hsp70, the rat monoclonal anti-Hsc70 and rabbit polyclonal HSF-1 antibodies purchased from Stressgen - Enzo Life Sciences, Inc., Plymouth Meeting, PA, USA. Anti-Bax rabbit polyclonal (N-20) and anti-VDAC-1 goat polyclonal (N-18) antibodies were purchased from Santa Cruz Biotechnology Inc., Santa Cruz, CA, USA. Anti-cytochrome $c$ mouse monoclonal antibody (556433) was obtained from BD Pharmingen Inc., San Diego, CA, USA. Anti-caspase 9 and -ERK antibodies were acquired from Cell Signaling Technology Inc., Danvers, MA, USA. The mouse monoclonal antibody against actin was obtained from Chemicon International Inc., Temecula, CA, USA. Anti-Bax 6 A7 monoclonal antibody and other reagents were purchased from Sigma-Aldrich, St. Louis, MO, USA.

Cell culture and viability assay. A549 and H358 lung cancer cell lines were cultured in Dulbecco's modified Eagle's medium containing $10 \%$ foetal bovine serum at $37^{\circ} \mathrm{C}$. BEAS-2B cells were grown in bronchial epithelial basal medium. All NSAID and cisplatin were dissolved in dimethyl sulphoxide and added to the medium at indicated concentrations. The activity of mitochondrial dehydrogenase 3-(4,5-dimethylthiazol-2-yl)-2,5-diphenyltetrazolium bromide (MTT) assay was used to measure cell death/survival. The reaction product was measured at $A_{570}$, and the relative viability of cells treated with reagents versus untreated cells was calculated.

TUNEL staining. The TUNEL assay was performed using an in situ cell death detection kit (F Hoffmann-La Roche, Basel, Switzerland) according to the manufacturer's instructions. The ratio of TUNEL-positive cells to the total number of cells was calculated.

Immunoprecipitation and cell fractionation. A549 cells were lysed in RIPA buffer ( $50 \mathrm{mM}$ Tris- $\mathrm{HCl}, \mathrm{pH} 7.5,150 \mathrm{mM} \mathrm{NaCl}, 1 \mathrm{mM}$ sodium orthovanadate, $1 \mathrm{mM}$ EDTA, $0.1 \%$ NP-40, $10 \mathrm{mM}$ NaF) containing the Calbiochem Protease Inhibitor Cocktail Set III (Merck KGaA, Darmstadt, Germany). The cell lysates and immunoprecipitates were resolved in Laemmli sample buffer. The samples underwent sodium dodecyl sulphate-polyacrylamide gel electrophoresis were transferred to a polyvinylidene difluoride membrane, reacted with the respective antibodies, and detected with an ECL chemiluminescence detection kit (GE Healthcare, Fairfield, CT, USA). For the immunoprecipitation, the cell lysates were 
incubated with the indicated antibodies for $1 \mathrm{~h}$ at $4{ }^{\circ} \mathrm{C}$. Protein G-sepharose beads were added to collect the immunocomplexes for an additional $1 \mathrm{~h}$ of incubation The pellets were washed three times with lysis buffer. The mitochondria and cytosol fractions were prepared as described previously. ${ }^{45}$

Chromatin immunoprecipitation (ChIP) assay. ChIP assays were performed as described previously, ${ }^{46}$ using an EZ ChIP kit (Upstate Biotechnology Inc., Waltham, MA, USA). Briefly, after adding formaldehyde, A549 cells were suspended in SDS lysis buffer and the chromatin DNA was disrupted by sonication. For the immunoprecipitation, the lysate was incubated with anti-HSF-1 antibody, followed by immobilization on salmon sperm DNA/Protein G agarose. The protein/DNA complexes extracted with elution buffer were heated to $65^{\circ} \mathrm{C}$ for $6 \mathrm{~h}$ to reverse cross-links, then digested with proteinase K. DNA fragments were amplified in PCR with the ChIP assay primers containing the heat shock element sites in human Hsp70 promoter. PCR primers for the ChIP assay were as follows: Hsp70 (-103/+7) (F) 5'-TGATTGGTCCAAGGAAGGCT-3' and (R) 5'-AAAAAGGTAGTGGACTGTCGC-3'.

Reverse transcriptase-PCR. RT-PCR was carried out, using a Qiagen (Valencia, CA, USA) One-Step RT-PCR Kit. We used the following primer pairs to amplify. Hsp70, (F) 5'-ATGAAGCACTGGCCTTTCCA-3', (R) 5'-TTGTTCTGGC TGATGTCCTT-3'; Hsc70, (F) 5'-TGGAACTATTGCTGGTCTCAA3'; (R) 5'-AG AACCACCAACCAGGACAAT-3'; HSF-1, (F) 5'-TTCGACCAGGGCCAGTTT-3'; (R) 5'-AGAGCTGGCCACAGCATCA-3'; actin, (F) 5'-AGAGGCATCCTCACCCTG A-3'; (R) $5^{\prime}$-CATCTCTTGCTCGAAGTCCA-3'. The products were examined by agarose gel electrophoresis after 23 cycles.

RNA interference. The sequences of the sense strands used to generate specific siRNA were obtained as follows: HSF-1, 5'-AAGTACTTCAAGCACAAC AA-3', 5'-AAGAGTGAAGACATAAAGAT-3', 5'-AAGTCGTCAACAAGCTCATT-3'. The siRNAs were synthesized using the Silencer siRNA construction kit (Ambion; Applied Biosystems Inc., Carlsbad, CA, USA). Double-stranded Hsp70 and control siRNA duplex were synthesized as followed by Qiagen: Hsp70-specific sequence, 5'-CCAUUGAGGAGGUAGAUUAdTdT-3'. A549 cells were transfected with each siRNA (10 nmol//) using the Lipofectamine 2000 (Invitrogen; Applied Biosystems Inc.), and grown for $72 \mathrm{~h}$ to allow an effective decrease in the expression of the respective target molecules.

Quantification of apoptosis by flow cytometry. A549 cells were washed with Annexin $V$ staining buffer (10 mM HEPES pH 7.4, $150 \mathrm{mM} \mathrm{NaCl}$, $5 \mathrm{mM} \mathrm{KCl}, 1 \mathrm{mM} \mathrm{MgCl}, 1.8 \mathrm{mM} \mathrm{CaCl}_{2}$ ), and incubated with CF488A-Annexin V and propidium iodide (Biotium, Inc., Hayward, CA, USA) in staining buffer for $30 \mathrm{~min}$ at $37^{\circ} \mathrm{C}$ in the dark. Fluorescence was measured using a FACSCalibur (BD Biosciences, San Jose, CA, USA), and the data were analyzed with CellQuest software (BD Biosciences).

JC-1 staining and quantification. A549 cells were cultured at $37^{\circ} \mathrm{C}$ for $48 \mathrm{~h}$ on glass chamber slides and treated with ibuprofen and cisplatin at the specified concentrations for $48 \mathrm{~h}$. Mitochondrial permeability transition was determined by staining the cells with $5,5^{\prime}, 6,6^{\prime}$-tetrachloro-1,1,3,3'-tetraethylbenzimidazolylcarbocyanin iodide (JC-1; Molecular Probes, Invitrogen, Carlsbad, CA, USA) in the dark. The cells were subsequently washed with assay buffer according to the manufacturer's protocol and immediately imaged using a fluorescence microscope (Keyence Corporation, Osaka, Japan) with the red ( $\lambda_{\text {excitation }}: 560 \pm 40 \mathrm{~nm}$ band pass filter, $\lambda_{\text {detection }}: 630 \pm 60 \mathrm{~nm}$ band pass filter) and green ( $\lambda_{\text {excitation }}: 470 \pm 40 \mathrm{~nm}$ band pass filter, $\lambda_{\text {detection }}: 535 \pm 50 \mathrm{~nm}$ band pass filter) fluorescence channels. Flow cytometric analysis was assayed with the JC-1 Mitochondrial Membrane Potential Kit (AAT Bioquest, Sunnyvale, CA, USA) according to the manufacturer's directions, using a FACSCalibur, and the results were analyzed by CellQuest software.

In vitro casapse-9 activity determination. Caspase-9 activity was measured by a fluorometric assay in whole-cell lysates using Ac-Leu-Glu-His-AspMCA substrate (Peptide International Inc., Louisville, KY, USA). A549 cell extracts were mixed with Ac-LEHD-MCA in ICE standard buffer (100 mM HEPES pH 7.5, $10 \%$ sucrose, $0.1 \%$ CHAPS, $10 \mathrm{mM}$ DTT, $1 \mathrm{mM}$ PMSF), and cleavage of the fluorogenic peptide substrate was monitored at $37^{\circ} \mathrm{C}$ for $30 \mathrm{~min}$ by a SPECTRA max GEMINI EM (Molecular Device, Sunnyvale, CA, USA) fluorometer with excitation at $370 \mathrm{~nm}$ and emission at $460 \mathrm{~nm}$.

\section{Conflict of Interest}

The authors declare no conflict of interest.

Acknowledgements. This work was supported, in part, by a Grant-in-Aid (23591477) from the Ministry of Education, Culture, Sports, Science, and Technology of Japan.

1. Tavaria M, Gabriele T, Kola I, Anderson RL. A hitchhiker's guide to the human Hsp70 family. Cell Stress Chaperones 1996; 1: 23-28.

2. Jaattela M. Escaping cell death: survival proteins in cancer. Exp Cell Res 1999; 248: 30-43.

3. Aghdassi A, Phillips P, Dudeja V, Dhaulakhandi D, Sharif R, Dawra R et al. Heat shock protein 70 increases tumorigenicity and inhibits apoptosis in pancreas adenocarcinoma. Cancer Res 2007; 67: 616-625.

4. Ciocca DR, Clark GM, Tandon AK, Fuqua SA, Welch WJ, McGuire WL. Heat shock protein hsp70 in patients with axillary lymph node-negative breast cancer. J Natl Cancer Inst 1993; 85: $570-574$.

5. Cornford PA, Dodson AR, Parsons KF, Desmond AD, Woolfenden A, Fordham M et al. Heat shock protein expression independently predicts clinical outcome in prostate cancer. Cancer Res 2000; 60: 7099-7105.

6. Vargas-Roig LM, Gago FE, Tello O, Aznar JC, Ciocca DR. Heat shock protein expression and drug resistance in breast cancer patients treated with induction chemotherapy. Int J Cancer 1998; 79: 468-475.

7. Igney $\mathrm{FH}, \mathrm{Krammer} \mathrm{PH}$. Death and anti-death: tumor resistance to apoptosis. Nat Rev Cancer 2002; 2: 277-288.

8. Gabai VL, Meriin AB, Mosser DD, Caron AW, Rits S, Shifrin VI et al. Hsp70 prevents activation of stress kinases. A novel pathway of cellular thermotolerance. $\mathrm{J}$ Biol Chem 1997: 272: 18033-18037.

9. Meriin AB, Yaglom JA, Gabai VL, Zon L, Ganiatsas S, Mosser DD et al. Protein-damaging stresses active c-Jun N-terminal kinase via inhibition of its dephosphorylation: a novel pathway controlled by Hsp72. Mol Cell Biol 1999; 19: 2547-2555.

10. Park HS, Cho SG, Kim GK, Hwang HS, Noh KT, Kim MS et al. Heat shock protein hsp72 is a negative regulator of apoptosis signal-regulating kinase 1. Mol Cell Biol 2002; 22: $7721-7730$

11. Mosser DD, Morimoto RI. Molecular chaperones and the stress of oncogenesis. Oncogene 2004; 23: 2907-2918.

12. Beere HM, Wolf BB, Cain K, Mosser DD, Mahboubi A, Kuwana T et al. Heat-shock protein 70 inhibits apoptosis by preventing recruitment of procaspase- 9 to the Apaf-1 apoptosome. Nat Cell Biol 2000; 2: 469-475.

13. Saleh A, Srinivasula SM, Balkir L, Robbins PD, Alnemri ES. Negative regulation of the Apaf-1 apoptosome by Hsp70. Nat Cell Biol 2000; 2: 476-483.

14. Zermati Y, Garrido C, Amsellem S, Fishelson S, Bouscary D, Valensi F et al. Caspase activation is required for terminal erythroid differentiation. J Exp Med 2001; 193: 247-254

15. Schmitt E, Gehrmann M, Brunet M, Multhoff G, Garrido C. Intracellular and extracellular functions of heat shock proteins: repercussions in cancer therapy. J Leu Biol 2007; 81: 15-27.

16. Gotoh T, Terada K, Oyadomari S, Mori M. Hsp70-DnaJ chaperone pair prevents nitric oxide- and CHOP-induced apoptosis by inhibiting translocation of Bax to mitochondria. Cell Death Differ 2004; 11: 390-402.

17. Stankiewicz AR, Lachapelle G, Foo CZ, Radicioni SM, Mosser DD. Hsp70 inhibits heatinduced apoptosis upstream of mitochondria by preventing Bax translocation. J Biol Chem 2005; 280: 38729-38739.

18. Hosokawa N, Hirayoshi K, Nakai A, Hosokawa Y, Marui N, Yoshida M et al. Flavonoids inhibit the expression of heat shock proteins. Cell Struct Funct 1990; 15: 393-401.

19. Yokota S, Kitahara M, Nagata K. Benzylidene lactam compound, KNK437, a novel inhibitor of acquisition of thermotolerance and heat shock protein induction in human colon carcinoma cells. Cancer Res 2000; 60: 2942-2948.

20. Westerheide SD, Kawahara TL, Orton K, Morimoto RI. Triptolide, an inhibitor of the human heat shock response that enhances stress-induced cell death. J Biol Chem 2006; 281 : 9616-9622.

21. Aghdassi A, Phillips P, Dudeja V, Dhaulakhandi D, Sharif R, Dawra R et al. Heat shock protein 70 increases tumorigenicity and inhibits apoptosis in pancreatic adenocarcinoma. Cancer Res 2007; 67: 616-625.

22. Phillips PA, Dudeja V, McCarroll JA, Borja-Cacho D, Dawra RK, Grizzle WE et al. Triptolide induces pancreatic cancer cell death via inhibition of heat shock protein 70. Cancer Res 2007; 67: 9407-9416.

23. Giardiello FM, Offerhaus GJ, DuBois RN. The role of nonsteroidal anti-inflammatory drugs in colorectal cancer prevention. Eur J Cancer 1995; 31A: 1071-1076.

24. Thun MJ, Henley SJ, Patrono C. Nonsteroidal anti-inflammatory drugs as anticance agents: mechanistic, pharmacologic, and clinical issues. J Natl Cancer Inst 2002; 94: 252-266

25. Smalley WE, DuBois RN. Colorectal cancer and nonsteroidal anti-inflammatory drugs. Adv Pharmacol 1997; 39: 1-20. 
26. Shiff SJ, Koutsos MI, Qiao L, Rigas B. Nonsteroidal antiinflammatory drugs inhibit the proliferation of colon adenocarcinoma cells: effects on cell cycle and apoptosis. Exp Cell Res 1996; 222: 179-188.

27. Klampfer L, Cammenga J, Wisniewski HG, Nimer SD. Sodium salicylate activates caspases and induces apoptosis of myeloid leukemia cell lines. Blood 1999; 93: 2386-2394.

28. Shiff SJ, Oiao L, Tsai LL, Rigas B. Sulindac sulfide, an aspirin-like compound, inhibits proliferation, causes cell cycle quiescence, and induces apoptosis in HT-29 colon adenocarcinoma cells. J Clin Invest 1995; 96: 491-503.

29. Yao M, Zhou W, Sangha S, Albert A, Chang AJ, Liu TC et al. Effect of nonselective cyclooxygenase inhibition with low-dose ibuprofen on tumor growth, angiogenesis, metastasis, and survival in a mouse model of colorectal cancer. Clin Cancer Res 2005; 11 : 1618-1628.

30. Zhang L, Yu J, Park BH, Kinzler KW, Vogelstein B. Role of BAX in the apoptotic response to anticancer agents. Science 2000; 290: 989-992.

31. Liou JY, Ghelani D, Yeh S, Wu KK. Nonsteroidal anti-inflammatory drugs induce colorectal cancer cell apoptosis by suppressing 14-3-3 $\varepsilon$. Cancer Res 2007; 67: 3185-3191.

32. Siddik ZH. Cisplatin: mode of cytotoxic action and molecular basis of resistance. Oncogene 2003; 22: 7265-7279.

33. Cho HJ, Kim JK, Kim KD, Yoon HK, Cho MY, Park YP et al. Upregulation of Bcl2 is associated with cisplatin-resistance via inhibition of Bax translocation in human bladder cancer cells. Cancer Lett 2006; 237: 56-66.

34. Bonay M, Soler P, Riquet M, Battesti JP, Hance AJ, Tazi A et al. Expression of heat shock proteins in human lung and lung cancers. Am J Respir Cell Mol Biol 1994; 10: 453-461.

35. Michils A, Redivo M, Zegers de Beyl V, de Maertelaer V, Jacobovitz D, Rocmans P et al. Increased expression of high but noy low molecular weight heat shock proteins in resectable lung carcinoma. Lung Cancer 2001; 33: 59-67.

36. Huang Q, Zu Y, Fu X, Wu T. Expression of heat shock protein 70 and 27 in non-small cell lung cancer and its clinical siginificance. J Huazhong Univ Sci Technolog Med Sci 2005; 25: 693-695.

37. Suzuki K, Ito Y, Wakai K, Kawado M, Hashimoto S, Seki N et al. Serum heat shock protein 70 levels and lung cancer risk: a case-control study nested in a large cohort studyt shock protein 70 levels and lung cancer risk: a case-control study nested in a large cohort study. Cancer Epidemiol Biomarkers Prev 2006; 15: 1733-1737.
38. Zimmermann M, Nickl S, Lambers C, Hacker S, Mitterbauer A, Hoetzenecker K et al. Discrimination of clinical stages in non-small cell lung cancer patients by serum HSP27 and HSP70: A multi-institutional case-control study. Clin Chim Acta 2012; 413: 1115-1120.

39. Singh U, Bonqcam-Rudloff E, Westermark B. A DNA sequence directed mutual transcription regulation of HSF1 and NFIX involves novel heat sensitive protein interactions. PLOS One 2009; 4: e5050.

40. Guo F, Rocha K, Bali P, Pranpat M, Fiskus W, Boyapalle S et al. Abrogation of heat shock protein 70 induction as a strategy to increase antileukemia activity of heat shock protein 90 inhibitor 17-allylamino-demethoxy geldanamycin. Cancer Res 2005; 65: 10536-10544.

41. Ren A, Yan G, You B, Sun J. Down-regulation of mammalian sterile 20-like kinase 1 by heat shock protein 70 mediates cisplatin resistance in prostate cancer cells. Cancer Res 2008; 68: 2266-2273.

42. Wang G, Reed E, Li QQ. Molecular basis of cellular response to cisplatin chemotherapy in non-small cell lung cancer. Oncol Rep 2004; 12: 955-965.

43. Juergens RA, Brahmer JR. Adjuvant treatment in non-small cell lung cancer: where are we now? J Natl Compr Canc Netw 2006; 4: 595-600.

44. Steel R, Doherty JP, Buzzard K, Clemons N, Hawkins CJ, Anderson RL. Hsp72 Inhibits Apoptosis Upstream of the Mitochondria and Not through Interactions with Apaf-1. J Biol Chem 2004; 279: 51490-51499.

45. Yano M, Nakamuta S, Shiota M, Endo H, Kido H. Gatekeeper role of 14-3-3 $\tau$ protein in HIV-1 gp120-mediated apoptosis of human endothelial cells by inactivation of Bad. AIDS 2007; 21: 911-920.

46. Yano M, Nakamuta S, Wu X, Okumura Y, Kido H. A novel function of 14-3-3 protein: $14-3-3 \zeta$ is a heat-shock-related molecular chaperone that dissolves thermal-aggregated proteins. Mol Biol Cell 2006; 17: 4769-4779.

cc) (1) (-) $\odot$ Cell Death and Disease is an open-access journal (c) licensed under a Creative Commons Attribution-NonCommercialNoDerivs 3.0 Unported License. To view a copy of this license, visit http://creativecommons.org/licenses/by-nc-nd/3.0/ 